\title{
MOLECULAR CHARACTERIZATION OF A MAIZE REGULATORY GENE
}

\section{Annual Progress Report}

\author{
Dr. Susan R. Wessler \\ University of Georgia Research Foundation, Inc. \\ Athens, GA 30602
}

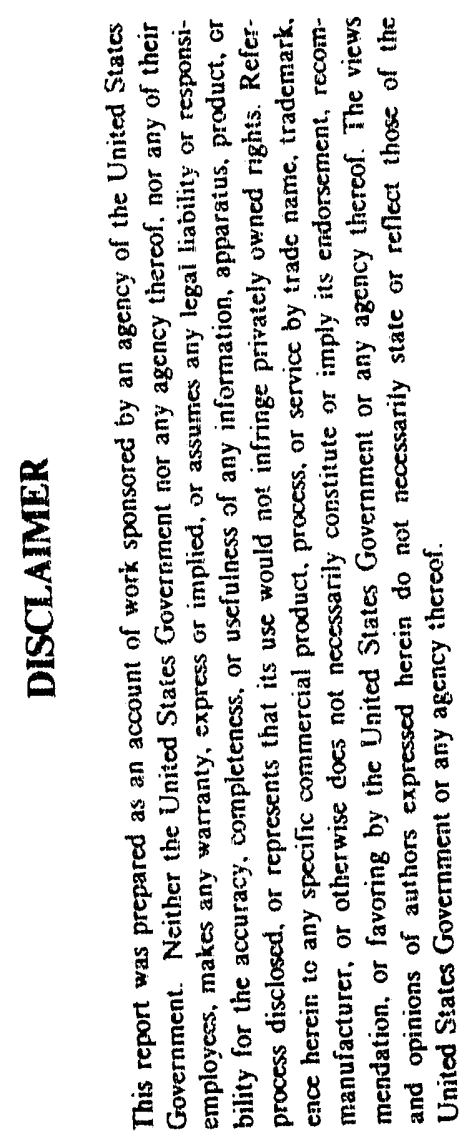

December 1991.

PREPARED FOR THE

U. S. DEPARTMENT OF ENERGY UNDER GRANT

DE-FG09.86ER13621 
Susan R. Wessler

\section{Progreen Report for Grant DE-FG09-86ER13621 \\ Report covers March 1990 through November 1991 Molecular Characterization of a Maize Regulatory Gene}

Previously Stated Objectives:

Three overall goals comprised the last competitive renewal proposal:

(1) biochemical characterization of the DNA binding properties of the $R$ protein.

(2) analysis of the $D s$ excision mutants of $R$-sc isolated by Jerry Kermicle

(Wisconsin)

(3) immunochemical analysis of the distribution of $\mathrm{R}$ protein in different $R$ alleles

Revised objectives:

The decision to revise the above aims is based on two factors: competition from Steve Goff in Vicki Chandler's lab (working on the $R$ homolog $B$ ) and new discoveries in my lab. Steve Goff has recently completed a beautiful study of the functional domains of the B protein. The Fromm and Chandler labs have collaborated to determine the importance of acidic and basic domains and have identified an additional domain that mediates interactions with the other anthocyanin regulatory protein, $\mathrm{C} 1$. Since we had yet to embark on this aspect of the project, no time was wasted duplicating their efforts. Vicki and I are close personal and professional friends and open lines of communication have and will continue to prevent wasteful duplication of effort.

Another reason for not pursuing DNA binding and domain swapping studies was our discovery that the $L c$ gene is regulated posttranscriptionally. Based on our initial bombardment studies we concluded that promoter diversity was responsible for the diversity of naturally occurring $R$ alleles (Ludwig et al.1989; Ludwig et al.1990). However, over the last funding period we have found that (1) $R$ is controlled at the level of translation initiation and (2) intron 1 is alternatively spliced. The experiments described in Sections I and II seek to quantify these effects and to determine whether they contribute to the tissue specific expression of select $R$ alleles. I have chosen to emphasize this aspect of the project. because very little is understood about the post-transcriptional regulation of plant genes. Section III and IV describe experiments designed to identify important structural components of the R protein. Again, our approach differs from the approach of the Chandler lab and is designed to complement their analysis rather than duplicate efforts.

I. Translational control of the Lc gene:

The $L c$ cDNA cloned and sequenced in my lab (Ludwig et al.1989) contains a $240 \mathrm{nt} 5$ leader region that contains three AUG codons upstream of the initiation codon of the Lc protein (Figure 1). Within this leader region is a 
38 codon minicistron that contains the 3 in frame upstream AUGs and is terminated by four in frame stop condons. Upstream AUGs have been implicated in the regulation of several animal genes (Kozak, 1989). There is only a single report of a plant gene regulated at this level and this is of a viral gene (Futterer et al.1990). The following experimental results led us to investigate the possibility that $R$ is regulated post-transcriptionally: (i) in vitro translation of the full length $L c$ cDNA in rabbit reticulocyte lysates proved extremely difficult until most of the 5 ' leader was deleted, (ii) particle bombardment experiments revealed that the expression of constructs containing the full-length $L c$ cDNA driven by the CaMV $35 \mathrm{~S}$ promoter induced 50 fold fewer red anthocyanin spots than constructs deleted for the 5 ' leader region (nt 1 through 195).

The inhibitory effect of the 5 leader region could result from the presence of the AUGs, secondary structure within this region and/or translation of the minicistron. To further investigate the regulatory role of this region, we have used site-directed mutagenesis to generated a series of point mutations in each of the upstream AUGs (Figure 2). These mutated $5^{\prime}$ leaders have been returned to their exact position within the $L c$ cDNA expression construct. To assess the effect of each and all mutations on $L c$ expression, we are carrying out cobombardment experiments where the Lc constructs are delivered with two other vectors, $\mathrm{pBz} 1 \mathrm{LUC}$ (obtained from $\mathrm{B}$. Roth, Pioneer) and pAdhCAT (obtained from S. Goff, Oregon). 'The former contains the firefly luciferase gene under the control of the $L c$-responsive $B z 1$ promoter. pAdhCAT is a control vector that is not influenced by the $L c$ protein. In this assay, luciferase levels provide a measure of the ability of $L c$ constructs to trans-activate the $B z I$ promoter. This approach is very similar to that used in other labs (Goff et al.1990; Roth et al.1991). Preliminary data indicates the following:

(i) Luciferase levels are increased 50 fold when nt 1-195 are deleted. (ii) Luciferase levels are increased about 60 fold (from wild-type leader levels) when the three upstream AUGs are mutated (mut123, Figure 2). This result suggests that ribosome binding rather than mRNA secondary structure is inhibitory. However, an inhibitory role for the putative 38aa minicistron product cannot be ruled out.

(iii) Luciferase levels intermediate between wild-type and mut 123 are seen with leaders containing one or two AUGs.

Future experiments will also assess whether the 38aa polypeptide is produced and whether it has a regulatory role.

II. Alternative splicing of $L c$ transcripts:

The second level of post-transcriptional control we are investigating is tissue specific alternative splicing of $L c$ mRNA. $L c$ mRNA isolated from female spiklets shows a characteristic broad band on Northern blots when probed with full-length cDNA (Ludwig et al.1989). When exon and intron specific probes are used we find that the broad band consists of 2 species; fully spliced and mRNA containing intron 1. Interestingly, intron 1 
contains 2 additional small open reading frames (see Figure 1) upstream of the Lc initiation codon. This means that a large fraction of $L c$ mRNA (about $50 \%$ based on Northern blots) contains 5 upstream AUGs. However, all reading frames have stop codons before the $L c$ initiation codon.

Over the next year we will test whether there is a tissue specific component to alternative splicing. For example, we will use Northern blots and PCR to determine whether the ratio of spliced to unspliced intron 1 varies in different tissues and, if so, whether there is also a correlation with pigmentation levels.

III. The $D_{s}$ derivatives of the $R$-sc allele:

Over $20 D$ s insertions into the $R$-sc transcription unit have been isolated by Jerry Kermicle. From each of these alleles he has isolated stable null, intermediate and revertant derivatives resulting from $D_{s}$ excision. These mutations represent an invaluable collection of very subtle mutations that have known effects on anthocyanin accumulation in the aleurone. We have begun to characterize these derivatives at two levels. First, we are using the particle bombardment assay described in Section I, above, to quantify the ability of each stable $D s$ derivative to transactivate the $B z 1$ promoter. Unlike the experiments described in Section I, where an in vitro mutagenized $R$ gene is cobombarded with the reporter construct, in these experiments we simply bombard the reporter construct (with the CAT control) into kernels containing a mutant $R$ gene and assess the endogenous $R$ proteing' ability to transactivate the $B z l$ promoter (as compared to wild-type and null controls). Second the stable mutations will be defined by sequencing the site of $D s$ excision following PCR amplification. Amino acid additions or other changes will then be correlated with expression of the mutant protein with respect to anthocyanin and luciferase levels.

We have begun by characterizing the R-sc:m 1 Ds 1 allele and 12 stable derivatives with null, intermediate and revertant phenotypes. Preliminary bombardment results show a good correlation between luciferase and anthocyanin levels, although some phenotypically null alleles result in low but significant luciferase pr oduction. The position of this $D s$ insertion has not been precisely determined but it is near the helix-loop-helix region. With this demonstration that the bombardment assay can be used to quantify the expression of endogenous mutant $R$ proteins, we will expand this study to other $D s$ derivatives. Of particular interest are $D s$ insertions in the DNA binding domain, in exon 1 and intron 1 (because of the potential regulatory effects described above) and in regions required for nuclear transport (see Section TV).

\section{Sequences responsible for nuclear targeting of the $R$ protein:}

Natashe Raikhel's lab is attempting to determine the amino acid domains of the $R$ protein that are required for its nuclear transport. The nuclear import of proteins larger than $50 \mathrm{kD}$ is mediated by specific nuclear localization sequences (NLS) within the proteins. There is no conserved 
sequence motifs for the NLS but they are known to be both necessary and sufficient for nuclear uptake. To determine specific NLS required for nuclear localization of the $75 \mathrm{kD} R$ protein, Mark Shieh, a student in the Raikhel lab, has fused all or part of $R$ to GUS to facilitate the localization of the protein within plant cells. The GUS-R gene fusions and the GUS gene were transiently expressed in tobacco suspension cells. Histrichemical \& lalysis of the tobacco cells revealed that the GUS-R fusion protein is ocalized to the nucleus. Additional experiments have identified three regions of the $R$ protein necessary for nuclear localization of GUS. One putative NLS is a domain in the carboxy terminus that is highly conserved in $R$ homologs from monocots and the dicot Antirrhinum.

With the characterization of NLS within the R protein continuing in Dr. Raikhel's lab, we have begun to search the collection of $D$ s alleles for insertions in these regions. We have identified two alleles that may have insertions in the carboxy terminus. Interestingly, both give rise to null and intermediate but not revertant derivatives. These derivatives will be characterized by the methods described in Section III.

\section{References:}

FUTTERER, J., K. GORDON, H. SANFACON, J. BONNEVILLE and T. HOHN, 1990 Positive and negative control of translation by the leader sequence of cauliflower mosaic virus pregenomic 35S RNA. EMBO J. 9:1697-1707.

GOFF, S.A., T.M. KLEIN, B.A. ROTH, et al, 1990 Transactivation of anthocyanin biosynthetic genes following transfer of B.regulatory benes into maize tissues. EMBO.J. 9:2517-2522.

KOZAK, M., 1989 The scanning model for translation: an update. J.Cell Biol. 108:229-241.

LUDWIG, S.R., B. BOWEN, L. BEACH and S.R. WESSLER, 1990 A regulatory gene as a novel visible marker for maize transformation. Science 247:449-450.

LUDWIG, S.R., L.F. HABERA, S.L. DELLAPORTA and S.R. WESSLER, $1989 L c$, a member of the maize $R$ gene family responsible for tissuespecific anthocyanin production, encodes a protein similar to transcriptional acitvators and contains the myc-homology region. Proc.Natl.Acad.Sci.USA. 86:7092-7096.

ROTH, B.A., S.A. GOFF', 'T.M. KLEIN and M.E. FROMM, 1991 C1dependent and $R$-dependent expression of the maize $B z I$ gene requires sequences with homology to mammalian $M y b$ and myc binding sites. Plant Cell 3:317-325. 
Susan R. Wessler, Grant \# DE-FG09-86ER13621
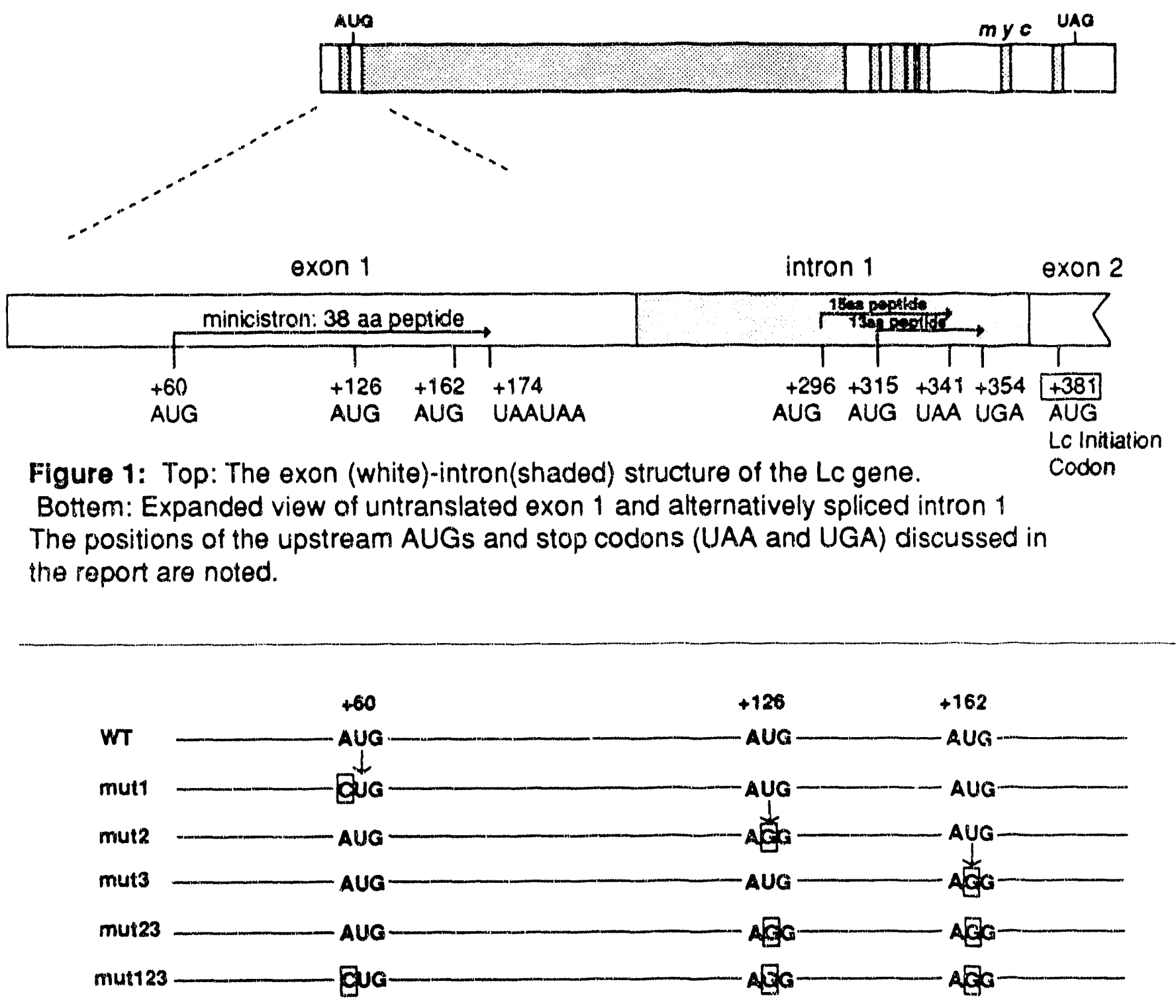

Figure 2: Site directed mutations of the 3 upstream AUGs in exon 1 generated in this study. 

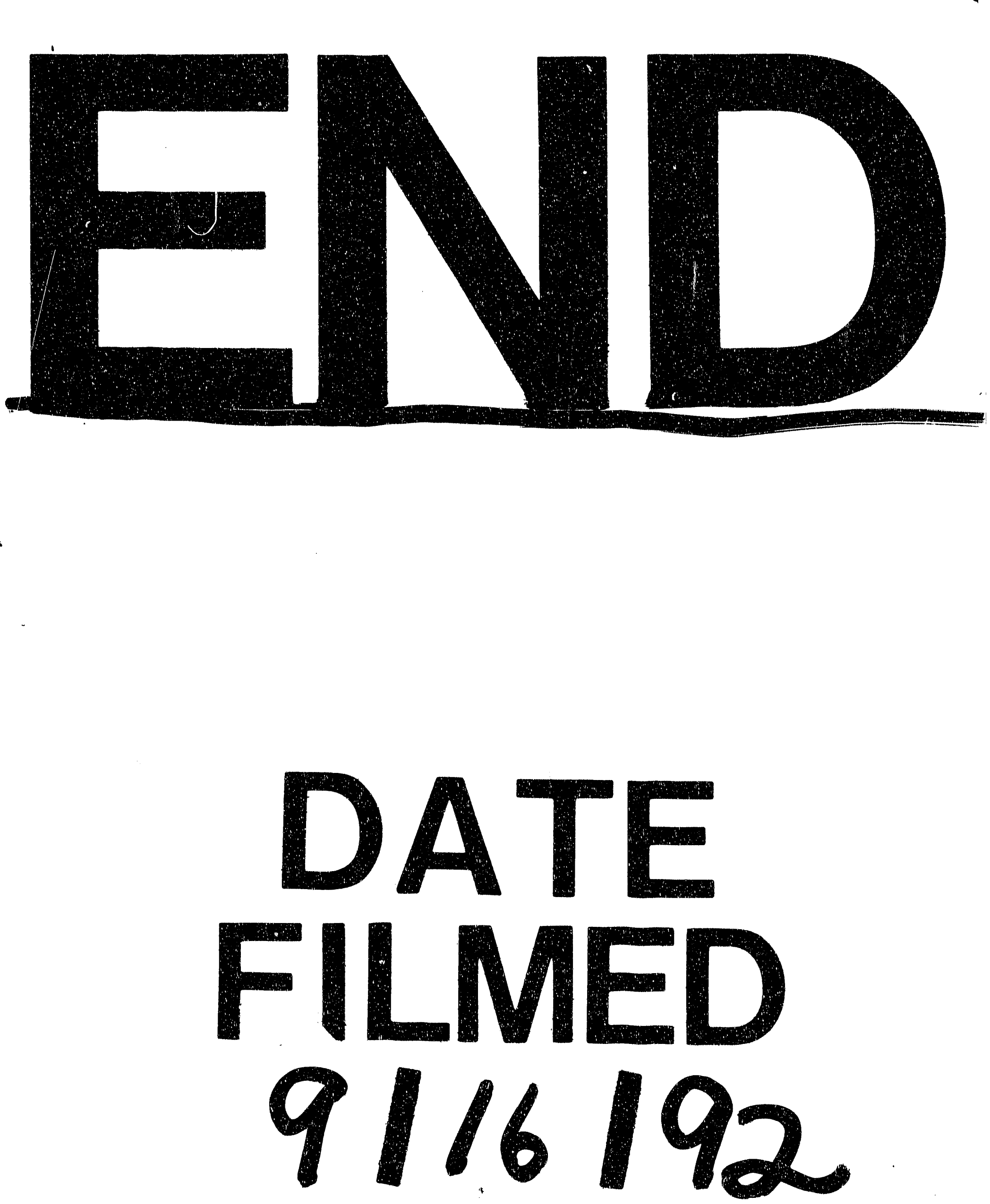


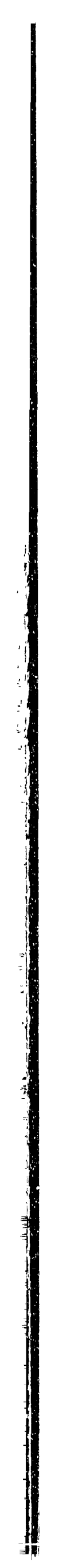

